\title{
Estudio de las Funciones de Coherencia de la Luz en un Sistema Jaynes-Cummings no Lineal
}

\section{Study of the Coherence Functions of Light in a Jaynes-Cummings non-linear System}

Yurimar Ruiz ${ }^{\mathrm{a}^{*}}$ Julio Gonzaléz ${ }^{b^{*}}$ Pablo Villamil ${ }^{\mathrm{c}^{*}}$

Fecha de Recepción: 08.11.2018

Fecha de Aceptación: 27.12.2018

DOI: https://doi.org/10.19053/01217488.v10.n1.2019.8098

\begin{abstract}
Resumen
En este trabajo se consideró un punto cuántico de dos niveles dentro de una cavidad con un medio no lineal tipo Kerr y un solo modo del campo electromagnético cuantizado. Se construyó la ecuación maestra considerando procesos disipativos, el término no lineal Kerr y se solucionó numéricamente para el estado estacionario teniendo en cuenta la temperatura. A partir de estos resultados se analizó la influencia que tiene el medio no lineal en la evolución temporal del número medio de fotones, la inversión de población, el espectro de fotoluminiscencia y se determinaron las características cuánticas-clásicas del estado de la luz mediante el cálculo de las funciones de coherencia de fotones de segundo orden.
\end{abstract}

Palabras clave: óptica no lineal, punto cuántico (QD), microcavidad, fotoluminiscencia (PL), ecuación maestra, funciones de correlación, Medio Kerr, estados cuánticos de luz.

\begin{abstract}
In this work, a two-level quantum dot inside of a cavity with a non-linear Kerr-type medium and a single mode of the electromagnetic field quantized was considered. The master equation was constructed taking into account dissipative processes, with the non-linear Kerr term, and it was numerically solved for the stationary state, taking into account the temperature. On the basis of these results, observable aspects of the state of the light were calculated, such as the average number of photons and the spectrum of photoluminescence, and the quantum-classical characteristics of the state of the light were determined by means of the calculation of the coherence functions of the second-order photons.
\end{abstract}

Key words: Nonlinear optics, quantum dot (QD), microcavity, photoluminescence (PL), master equation, Kerr medium, Quantum states of light.

a Departamento de Física, Universidad de Sucre, Sincelejo, Colombia.

* Autor de correspondencia: Yurimar86@gmail.com

b Departamento de Física, Universidad de Sucre, Sincelejo, Colombia.

* juliocg@ifi.unicamp.br

c Departamento de Física, Universidad de Sucre, Sincelejo, Colombia.

* pabloe1954@yahoo.es 


\section{INTRODUCCIÓN}

Desde un punto de vista teórico el problema más simple a considerar es un átomo aproximado a un sistema de dos niveles interactuando con un campo electromagnético cuantizado de un solo modo. El modelo de Jaynes-Cummings (MJC) es uno de los pocos modelos cuánticos exactamente solubles; este modelo ha atraído la atención de investigadores por más de 20 años y ha servido como un campo de pruebas para los modelos básicos de la interacción radiaciónmateria $[1,2]$. Este modelo es generalizado en muchas direcciones, ya que predice muchos efectos cuánticos novedosos que pueden ser verificados en experimentos de la física moderna [3-5]. La introducción del medio Kerr (material que al interactuar con radiación electromágnetica presenta un comportamiento no lineal) en el Hamiltoniano del sistema crea efectos no lineales. Una de las muchas aplicaciones de estos efectos no lineales es producir estados entrelazados, que se aplica ampliamente en información y comunicación cuántica [6-10]. En el trabajo de Joshi et. al., [11] se estudió el efecto de un medio Kerr en los fenómenos de colapso y reavivamiento en la inversión de población para un sistema de dos niveles. Wei et.al [12], investigaron el papel que juega el término no lineal en la modulación y preparación de estados cuánticos, proponiendo éste como un parámetro controlable en las investigaciones experimentales de este campo. E. del Valle et.al [13], estudiaron las correlaciones entre $\mathrm{N}$ fotones usando la teoría de frecuencia filtrada y tiempo resuelto. Presentando un método para calcular de manera eficiente las correlaciones entre un número arbitrario de fotones de frecuencias dadas y retardos de tiempo. Por otra parte, investigaron la emisión de estados desde el punto de vista de las correlaciones de dos fotones resueltos en frecuencia. Esto lo derivaron de un filtrado espectral, que se puede lograr mediante el uso de una cavidad o mediante la colocación de una serie de filtros de interferencia antes de los detectores. También estudiaron los llamados "procesos de salto" donde el sistema experimenta una transición directa de dos fotones [14]. El objetivo de este trabajo es estudiar el estado cuántico de la luz en un sistema Jaynes-
Cummings no lineal y disipativo modelado con una aproximación markoviana usando funciones de coherencia de segundo orden. En los procesos disipativos es importante considerar los efectos de la temperatura, ya que, estos inciden en los diferentes procesos cuánticos del sistema [1518] . Para calcular estas funciones de coherencia de segundo orden es necesario el cálculo de la función de correlación de primer y segundo orden.

\section{HAMILTONIANO DEL SISTEMA Y ECUACIÓN MAESTRA}

El modelo bajo consideración consta de un solo punto cuántico (QD) de dos niveles en un campo electromagnético cuantizado de un solo modo, ro-deado por un medio Kerr (ver figura 1).

El Hamiltoniano que describe este sistema viene dado por [7] :

$\hat{H}_{K}=\frac{1}{2} \hbar \omega_{a} \hat{\sigma}_{z}+\hbar \omega \hat{a}^{\dagger} \hat{a}+\hbar g\left(\hat{\sigma}_{+} \hat{a}+\hat{a}^{\dagger} \hat{\sigma}_{-}\right)+\chi \hat{a}^{\dagger 2} \hat{a}^{2}$,

donde el primer término del Hamiltoniano $\mathrm{H}^{\wedge} \mathrm{K}$ está asociado al QD libre, el segundo término hace referencia al modo cuantizado de la cavidad, el tercero a la interacción Jaynes-Cummings del QD con el modo cuantizado de la cavidad y el cuarto termino a la interacción del medio kerr con el modo cuantizado de la cavidad. $\hat{\sigma}_{z}$ es el operador de Pauli en la dirección z, $\hat{\sigma}_{+}, \hat{\sigma}-$ son los operadores de transición atómica de Pauli representados en una base $|g\rangle_{\text {y }}|e\rangle$ por matrices de Pauli, â y ât son los operadores aniquilación y creación del modo del campo de la cavidad, $\omega_{a}$ y $\omega$ son las frecuencias del QD y del modo de la cavidad respectivamente y g es la constante de acoplamiento Jaynes-Cummings. $\chi$ da cuenta de la dispersión de la no linealidad del medio Kerr . El medio Kerr se describe considerando a la cavidad como dos átomos de Rydberg, uno de ellos se comporta como un átomo de dos niveles que realiza una transición de dos fotones y el otro como un oscilador anármonico de frecuencia $\omega_{a}$ [11].

Los estados propios de $\hat{H}_{K}$ son los estados vestidos que cumplen $[19,20]$ : 


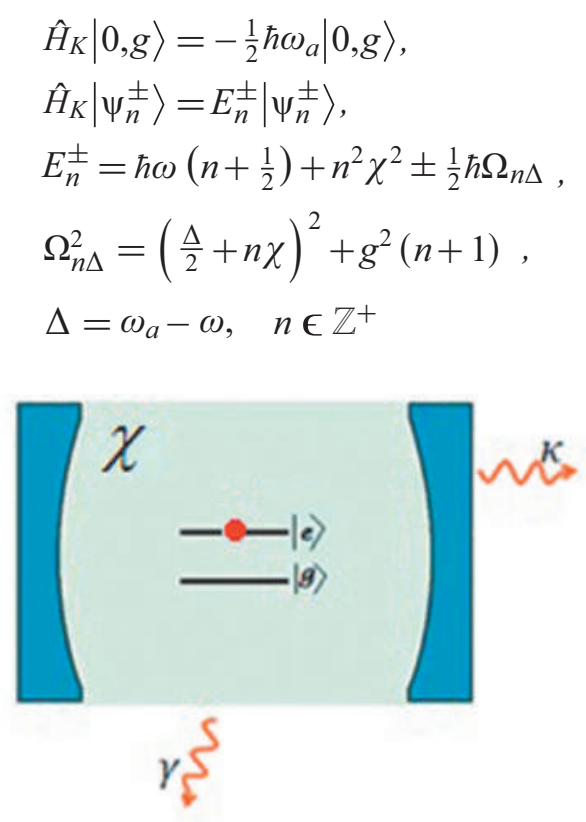

Figura 1. Representación esquemática el sistema estudiado

$\left|\psi_{n}^{ \pm}\right\rangle=\left(\begin{array}{c}\operatorname{sen} \theta_{n} \\ \cos \theta_{n}\end{array}\right)|n+1, g\rangle+\left(\begin{array}{c}\cos \theta_{n} \\ -\operatorname{sen} \theta_{n}\end{array}\right)|n, e\rangle$

$\tan \theta_{n}=\frac{g \sqrt{n+1}}{\Omega_{n \triangle}+\left(\frac{\Delta}{2}+n \chi\right)} \mid$

La evolución temporal de los estados puede expresarse como:

$e^{\frac{-i \hat{H} t}{\hbar}}|n, e\rangle=A_{n e}(t)|n, e\rangle+B_{n e}(t)|n+1, g\rangle$,

$e^{\frac{-i \hat{H} t}{\hbar}}|n+1, g\rangle=A_{n+1, g}(t)|n+1, g\rangle+B_{n+1, g}(t)|n, e\rangle$.

Las funciones de correlación excitonica y fotónica de primer y segundo orden en la imagen de Heisenberg están definidas como:

$G^{(1)}(t, \tau)=\left\langle\hat{P}^{\dagger}(t+\tau) \hat{P}^{-}(t)\right\rangle, \quad \hat{P}=\hat{\sigma}, \hat{a}$

$G^{(2)}(t, \tau)=\left\langle\hat{P}^{\dagger}(t) \hat{P}^{\dagger}(t+\tau) \hat{P}^{-}(t+\tau) \hat{P}^{-}(t)\right\rangle$

Considerando la evolución temporal de los estados para el MJC con medio kerr y asumiendo inicialmente el QD en el estado excitado y el campo en un estado de Fock, es decir $|n, e\rangle$ se encontró analíticamente [21 - 22] :

$G_{X}^{(1)}(t, \tau)=A_{n e}^{*}(t+\tau) A_{n e}(t) A_{n g}(\tau)$

$G_{X}^{(2)}(t, \tau)=\left|A_{n e}(t)\right|^{2}\left|B_{n g}(\tau)\right|^{2}$

$$
\begin{gathered}
G_{C}^{(1)}(t, \tau)=A_{n e}^{*}(t+\tau) A_{n-1, e}(\tau) A_{n e}(t) n \\
+B_{n e}^{*}(t+\tau) B_{n-1, e}(\tau) A_{n e}(t) \sqrt{n(n+1)} \\
+B_{n e}^{*}(t+\tau) B_{n g}(\tau) B_{n e}(t)(n+1) \\
G_{C}^{(2)}(t, \tau)=\left|A_{n e}(t)\right|^{2}\left|A_{n-1, e}(\tau)\right|^{2} n(n-1) \\
+A_{n e}^{*}(t) A_{n-1, e}^{*}(\tau) B_{n e}(t) B_{n g}(\tau) \sqrt{n(n+1)}(n-1) \\
+\left|A_{n e}(t)\right|^{2}\left|B_{n-1, e}(\tau)\right|^{2} n^{2}+A_{n e}^{*}(t) B_{n-1, e}^{*}(\tau) B_{n e}(t) \\
A_{n g}(\tau) n \sqrt{n(n+1)}+B_{n e}^{*}(t) A_{n, g}^{*}(\tau) A_{n e}(t) B_{n-1, e}(\tau) \\
n \sqrt{n(n+1)}\left|B_{n e}(t)\right|^{2} B_{n, g}^{*}(\tau) A_{n g}(\tau) n(n+1) \\
+B_{n e}^{*}(t) A_{n, g}^{*}(\tau) A_{n e}(t) B_{n-1, e}(\tau) n \sqrt{n(n+1)} \\
+\left|B_{n e}(t)\right|^{2} B_{n, g}^{*}(\tau) A_{n g}(\tau) n(n+1)
\end{gathered}
$$

donde:

$$
\begin{aligned}
A_{n e}(t) & =e^{\frac{-i E_{n}^{+} t}{\hbar}} \cos \theta_{n}^{2}+e^{\frac{-i E_{n}^{-} t}{\hbar}} \operatorname{sen} \theta_{n}^{2} \\
A_{n+1, g}(t) & =e^{\frac{-i E_{n}^{+} t}{\hbar}} \operatorname{sen} \theta_{n}^{2}+e^{\frac{-i E_{n}^{-} t}{\hbar}} \cos \theta_{n}^{2} \\
B_{n e}(t) & =\left(e^{\frac{-i E_{w}^{+} t}{\hbar}}-e^{\frac{-i E_{n}^{-} t}{\hbar}}\right) \operatorname{sen} \theta_{n} \cos \theta_{n}
\end{aligned}
$$

y

$$
\begin{aligned}
& A_{0 g}(t)=e^{\frac{i E_{0} t}{2 \hbar}}, \quad B_{0 g}(t)=0, \\
& B_{n e}(t)=B_{n+1, g}(t) .
\end{aligned}
$$

Con la función de correlación de primer orden se puede calcular el espectro para estados estacionarios, los cuales serán considerados en este trabajo [23 - 25]:

$$
S(w)=\frac{1}{\pi} \operatorname{Re} \int_{0}^{\infty} e^{i w \tau} G^{(1)}(t, \tau) d \tau
$$

El cálculo de la función de correlación de segundo orden nos permite encontrar la función de coherencia de segundo orden. Para la luz cuántica, la función de coherencia de fotones de segundo orden está dada por:

$$
g^{(2)}(\tau)=\frac{\left\langle\hat{a}_{1}^{\dagger}(t) \hat{a}_{2}^{\dagger}(t+\tau) \hat{a}_{2}(t+\tau) \hat{a}_{1}(t)\right\rangle}{\left\langle\hat{a}_{1}^{\dagger}(t) \hat{a}_{1}(t)\right\rangle\left\langle\hat{a}_{2}^{\dagger}(t+\tau) \hat{a}_{2}(t+\tau)\right\rangle}
$$

donde $\hat{a}_{i}$ representa la aniquilación de un fotón por la detección del detector $\mathrm{i}=1,2$ además el orden normal de los operadores del campo debe preservarse. 


\section{METODOLOGÍA}

Este trabajo consiste en investigar la dinámica de un sistema de dos niveles con un medio no lineal tipo kerr teniendo en cuenta efectos de temperatura. Este sistema se solucionó sin disipación para el modelo de Jaynes Cummings con medio kerr usando funciones de estado y numéricamente con disipación usando el formalismo de la ecuación maestra. La ecuación maestra que se utilizó para el cálculo numérico fue [26]:

$$
\begin{aligned}
\dot{\hat{\rho}}=\frac{1}{i \hbar}\left[\hat{H}_{K}, \hat{\rho}\right]+\frac{\kappa}{2}\left(1+n_{t h}\right)\left(2 \hat{a} \hat{\rho} \hat{a}^{\dagger}-\hat{a}^{\dagger} \hat{a} \hat{\rho}-\hat{\rho} \hat{a}^{\dagger} \hat{a}\right) \\
+\frac{\kappa}{2} n_{t h}\left(2 \hat{a}^{\dagger} \hat{\rho} \hat{a}-\hat{a} \hat{a}^{\dagger} \hat{\rho}-\hat{\rho} \hat{a} \hat{a}^{\dagger}\right) \\
+\frac{\gamma}{2}\left(2 \hat{\sigma}_{-} \hat{\rho} \hat{\sigma}_{+}-\hat{\sigma}_{+} \hat{\sigma}_{-} \hat{\rho}-\hat{\rho} \hat{\sigma}_{+} \hat{\sigma}_{-}\right)
\end{aligned}
$$

Ésta ecuación contiene cuatro términos: el primero describe la evolución unitaria del sistema QD-Cavidad, el segundo y tercer término describen el decaimiento de la cavidad y el cuarto término describe procesos de emisión espontánea del QD, $n_{t h}$ es el número medio de fotones del reservatorio térmico, $\kappa$ es la taza a la cual escapan fotones a través de los espejos de la cavidad y $\gamma$ es la taza de decaimiento debido a emisión espontánea del QD.

Al cambiar los parámetros libres en el modelo de nuestro sistema de estudio, $\kappa$ y $\gamma$ se pueden obtener dos regímenes diferentes de la dinámica: acoplamiento débil y acoplamiento fuerte entre el modo cuantizado de la cavidad y el qubit. Las escalas de tiempo características asociadas con cada proceso son: $\tau_{g}=\frac{1}{g}$, es la escala de tiempo de la interacción, $\tau_{\gamma}=\frac{1}{\gamma}$ es la escala de tiempo de emisión espontánea y $\tau_{\kappa}=\frac{1}{\kappa}$ es la escala de tiempo de disipación. Si $\mathrm{g}>>\kappa$, los fotones se almacenan de manera eficiente en la cavidad un tiempo $\tau_{\kappa}>>\tau_{g}$, así el campo y el átomo en la cavidad intercambian energía muchas veces antes de que el sistema entre en equilibrio. Cuando se cumple se dice que está en el régimen de acoplamiento fuerte. Si $\tau_{\kappa}<<\tau_{g}$ se dice que el sistema se encuentra en el régimen de acoplamiento débil $\mathrm{y}$ en este caso los fotones escapan de la cavidad antes de que puedan ser absorbidos por el qubit. El régimen donde se realizaron estos cálculos fue el régimen de acoplamiento fuerte y para simplificar el problema asumimos que la cavidad se encuentra en resonancia con el qubit, es decir, $\omega_{a}=\omega$ y además usamos $\kappa<\gamma$.

\section{RESULTADOS Y DISCUSIÓN}

A continuación se muestran los resultados obtenidos de los cálculos realizados: número promedio de fotones, número medio de quantos de energía asociados al punto cuántico $\left\langle\hat{\sigma}^{\dagger} \hat{\sigma}\right\rangle$ en función de $t$, espectro de fotoluminiscencia fotónico y función de coherencia de segundo orden para el MJC con y sin medio kerr. Para el número promedio de fotones $\left\langle a^{\wedge \dagger} a^{\wedge}\right\rangle$ y el valor esperado $\left\langle\hat{\sigma}^{\dagger} \hat{\sigma}\right\rangle$ en función de $t$ se hizo el estudio con y sin disipación. Para el espectro de fotoluminiscencia fotónico y función de coherencia de segundo orden se hizo con disipación, ya que se trabajó con estados estacionarios. La condición inicial que se consideró al momento de realizar los cálculos fue $|e, 1\rangle$, lo que indica que se asumió el QD en el estado 1 excitado y un fotón en la cavidad. En los sistemas donde se consideró la disipación las gráficas se realizaron para número medio de fotones en el reservorio $n_{t h} \geq 0$.

La Figura 2 corresponde a la gráfica del número promedio de fotones y el valor esperado $\left\langle\sigma^{\wedge} \sigma^{\wedge}\right\rangle$ en función del tiempo sin disipación para dos casos concretos. El primero corresponde al caso donde el medio es lineal, es decir, MJC sin efecto kerr $(\chi=0)$. El segundo caso incluye la parte no lineal $(\chi \neq 0)$. De la figura se observa un comportamiento oscilatorio y periódico para ambos casos. De estas cantidades físicamente lo que está sucediendo en el sistema es que hay un fotón en la cavidad y el QD está en el estado excitado, al momento de que el QD pasa al estado más bajo, la cavidad gana un fotón quedando ésta con dos fotones como se ve en la figura y el QD en el estado base y así sucesivamente vuelve el QD absorbe un fotón y pasa al estado excitado. Se puede observar que al agregar la parte del medio kerr para diferentes valores del parámetro $\chi$, la amplitud de las oscilaciones se reduce a medida que el valor de $\chi$ se hace más grande, de modo que la frecuencia de oscilación aumenta.

En la Figura 3 se puede observar la evolución temporal del número promedio de fotones, para 
valores $n_{t h}=0$ (a), $n_{t h}=1$ (b), $n_{t h}=2$ (c), y $n_{t h}=3$ (d) y para diferentes valores de $\chi$. De la gráfica vemos un comportamiento oscilatorio de dicho parámetro, el cual presenta pérdidas. En cada gráfica se puede observar que al aumentar $\chi$, la amplitud de las oscilaciones se reduce, debido principalmente a la taza de pérdidas a través de la cavidad. También se puede observar que las gráficas 3(b), 3(c) y 3(d) convergen aproximadamente al valor del número térmico de fotones, lo que significa que hay un intercambio de energía entre la cavidad y el reservorio hasta alcanzar el equilibrio térmico.
En las Figuras 4 se puede observar el valor esperado de $\left\langle\hat{\sigma}^{\dagger} \hat{\sigma}\right\rangle$ en función de tiempo $t$ del MJC, para $n_{t h}=0(\mathrm{a}), n_{t h}=1(\mathrm{~b}), n_{t h}=2(\mathrm{c}), \mathrm{y} n_{t h}=3(\mathrm{~d})$ y para diferentes valores de $\chi$. Se evidencia un comportamiento oscilatorio y con pérdidas, reduciendose la amplitud de las oscilaciones a medida que el valor de $\chi$ se hace más grande. El decrecimiento de $\left\langle\hat{\sigma}^{\dagger} \hat{\sigma}\right\rangle$ es debido principalmente a la emisión espontánea del QD. También se pueden observar oscilaciones de Rabi que tienden a amortiguarse o colapsar. Las figuras 4(b), 4(c) y 4(d) se interceptan en un punto, debido a que el QD trata de ponerse en equilibrio térmico con el ambiente.
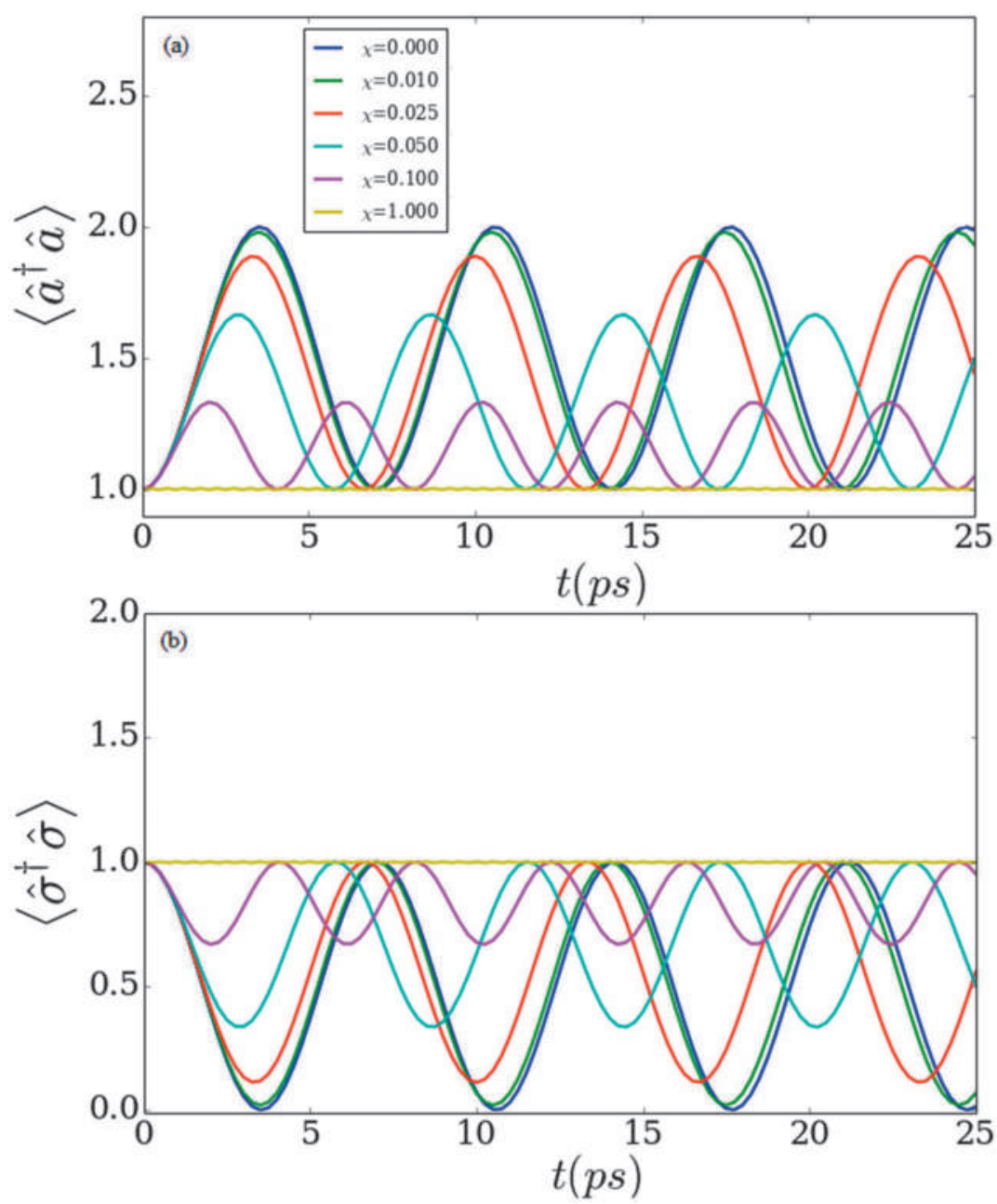

Figura 2. Evolución temporal del número promedio de fotones y el valor esperado de $\left\langle\hat{\sigma}^{\dagger} \hat{\sigma}\right\rangle$ sin disipación, $\frac{\omega}{2 \pi}=1,0 G H z$, $\frac{\omega_{a}}{2 \pi}=1,0 \mathrm{GHz}, \frac{g}{2 \pi}=0,05 \mathrm{GHz}$, para diferentes valores de $\chi$. 

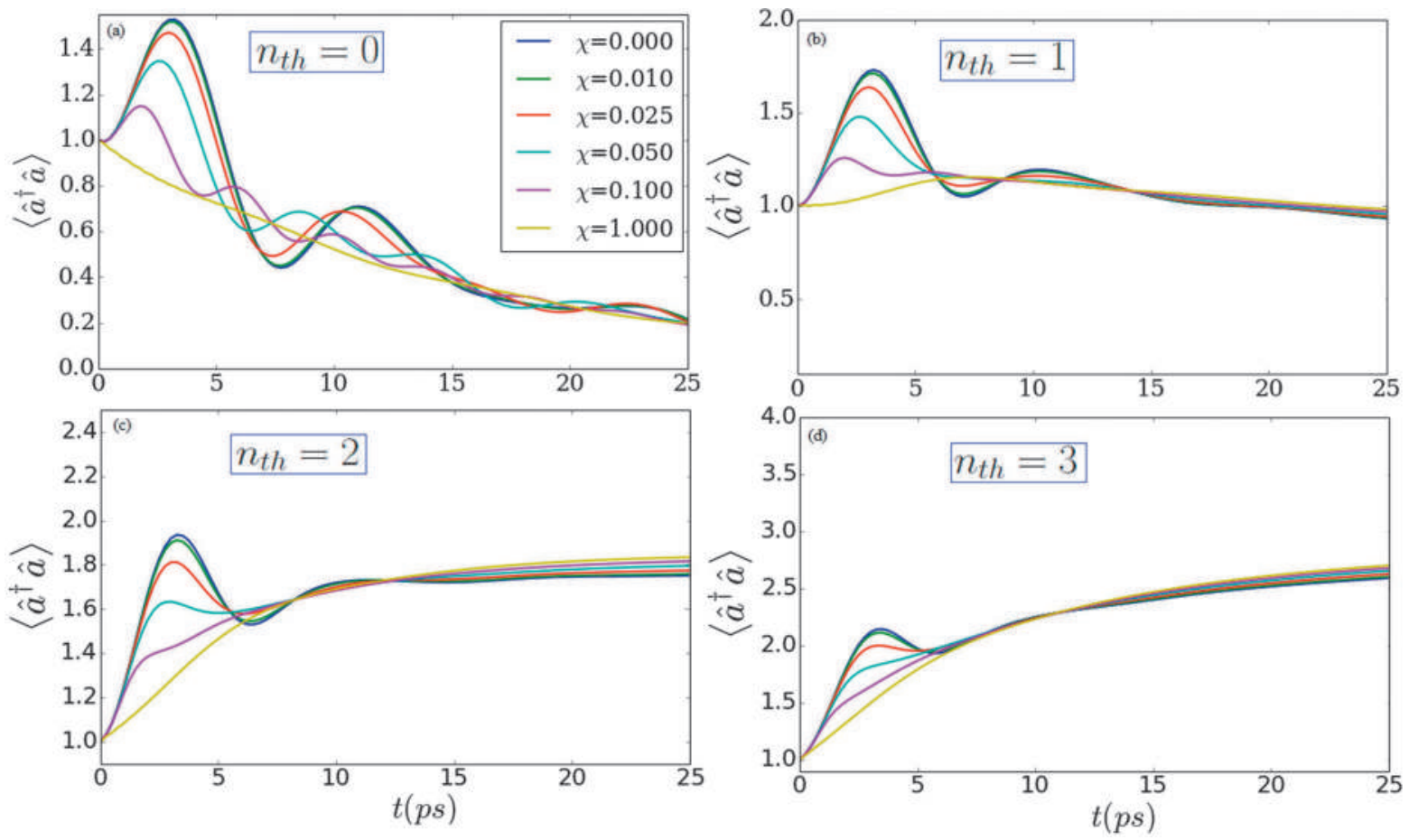

Figura 3. Evolución temporal del número promedio de fotones con disipación, $\frac{\omega}{2 \pi}=1,0 G H z, \frac{\omega_{a}}{2 \pi}=1,0 G H z, \frac{g}{2 \pi}=0,05 G H z$, $\kappa=0,08 G H z, \gamma=0,05 G H z$, (a) $n_{t h}=0$, (b) $n_{t h}=1$, (c) $n_{t h}=2$, (d) $n_{t h}=3$, para diferentes valores de $\chi$.
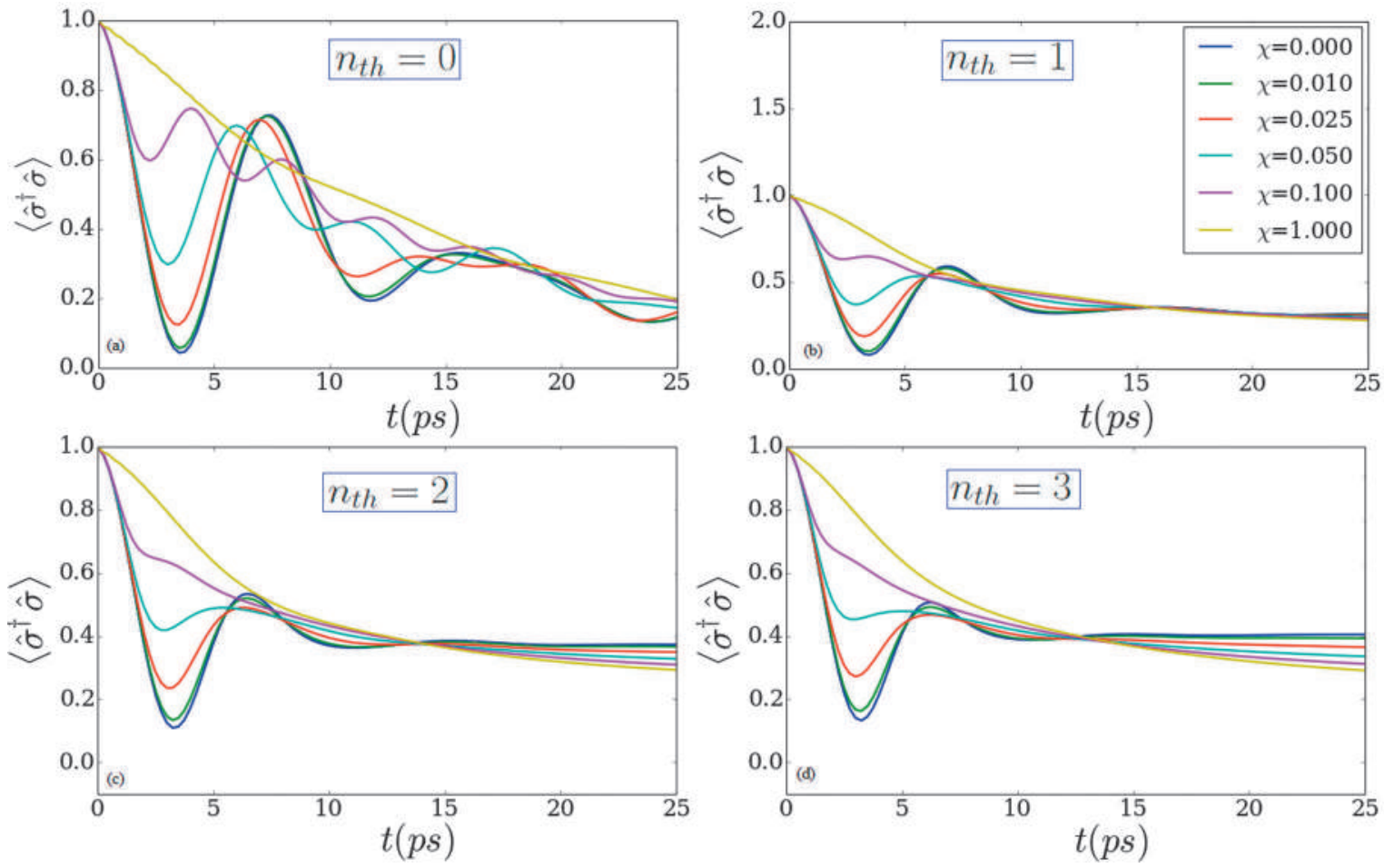

Figura 4. Valor esperado de $\left\langle\hat{\sigma}^{\dagger} \hat{\sigma}\right\rangle$ en función de $t$ con disipación $\frac{\omega}{2 \pi}=1,0 G H z, \frac{\omega_{a}}{2 \pi}=1,0 G H z, \frac{g}{2 \pi}=0,05 G H z, \kappa=$ $0,08 G H z, \gamma=0,05 G H z$ (a) $n_{t h}=0$, (b) $n_{t h}=1$, (c) $n_{t h}=2$, (d) $n_{t h}=3$, para diferentes valores de $\chi$.

En la Figura 5 se muestra el espectro de energía en función de la frecuencia para $n_{t h}=0$. El espectro obtenido presenta dos máximos en frecuencia y es simétrico con respecto al valor de la frecuencia de la cavidad. Vemos que no hay un cambio significativo a medida que el valor de $\chi$ 
aumenta. En la figura 6 se muestra el espectro de energía en función de la frecuencia para $n_{t h}=1(a)$, $n_{t h}=2(b)$ y $n_{t h}=3(c)$. A la derecha se muestra una gráfica ampliada de la misma para poder ver mejor la región donde $S(w) \neq 0$. La introducción de la nolinealidad y el $n_{t h}$ causa cambios drásticos en el espectro. En las figuras 6(a), 6(b) y 6(c), para $\chi=0$, el espectro sigue siendo simétrico para el valor de la frecuencia de la cavidad, pero para $\chi \neq 0$ los espectros son asimétricos. También se observa que el efecto de la temperatura para $\chi=0$ lleva cambios en la forma de la línea. Los picos se mueven uno hacia el otro tratando de fusionarse en un solo pico. Se puede observar en la Figura 6(a) que a medida que $\chi$ se hace más grande la amplitud de las oscilaciones disminuye y se tratan de formar más picos, sin embargo, en las Figuras 6(b) y 6(c) esos picos van desapareciendo a medida que el valor de $n_{t h}$ aumenta.

En la Figura 7 se muestra la función de coherencia de segundo orden como función $\tau$ para (a) $n_{t h}=0,(b) n_{t h}=1$, (c) $n_{t h}=2$ y (d) $n_{t h}=3$ y para diferentes valores de $\chi$. La función $g^{(2)}(\tau)$ mide la probabilidad de coincidencia de dos fotones que se detectan con una diferencia de tiempo. Típicamente esta función de coherencia de segundo orden se utiliza para encontrar el carácter estadístico de las fluctuaciones de la intensidad. Observamos que el mínimo de $g^{(2)}(\tau)$ para (a) $n_{\text {th }}$ $=0$, ocurre en $\tau=0$ y es periódica cada $\tau=10$ ps, sin embargo, el mínimo de $g^{(2)}(\tau)$ para $(b)$ $n_{t h}=1$, (c) $n_{t h}=2$, (d) $n_{t h}=3$, solo se da en $\tau=0$. Además $g^{(2)}(\tau)>g^{(2)}(0)$ para $\tau>0$ para los cuatro casos dados anteriormente, indicando que la luz es antiagrupada. Por otra parte, la estadística de fotones de la luz es Poissoniana para todos los casos. Observamos que los dos fenómenos vistos son netamente cuánticos, es decir, no hay análogo clásico para este sistema en estudio. En cada gráfica se puede observar que al agregar la parte del medio kerr para diferentes $\chi$, la amplitud de las oscilaciones cambia a medida que el valor de $\chi$ aumenta. También se puede observar que a medida que $n_{t h}$ aumenta $g^{(2)}(\tau) \rightarrow 1$ cuando $\tau \rightarrow$ $\infty$.

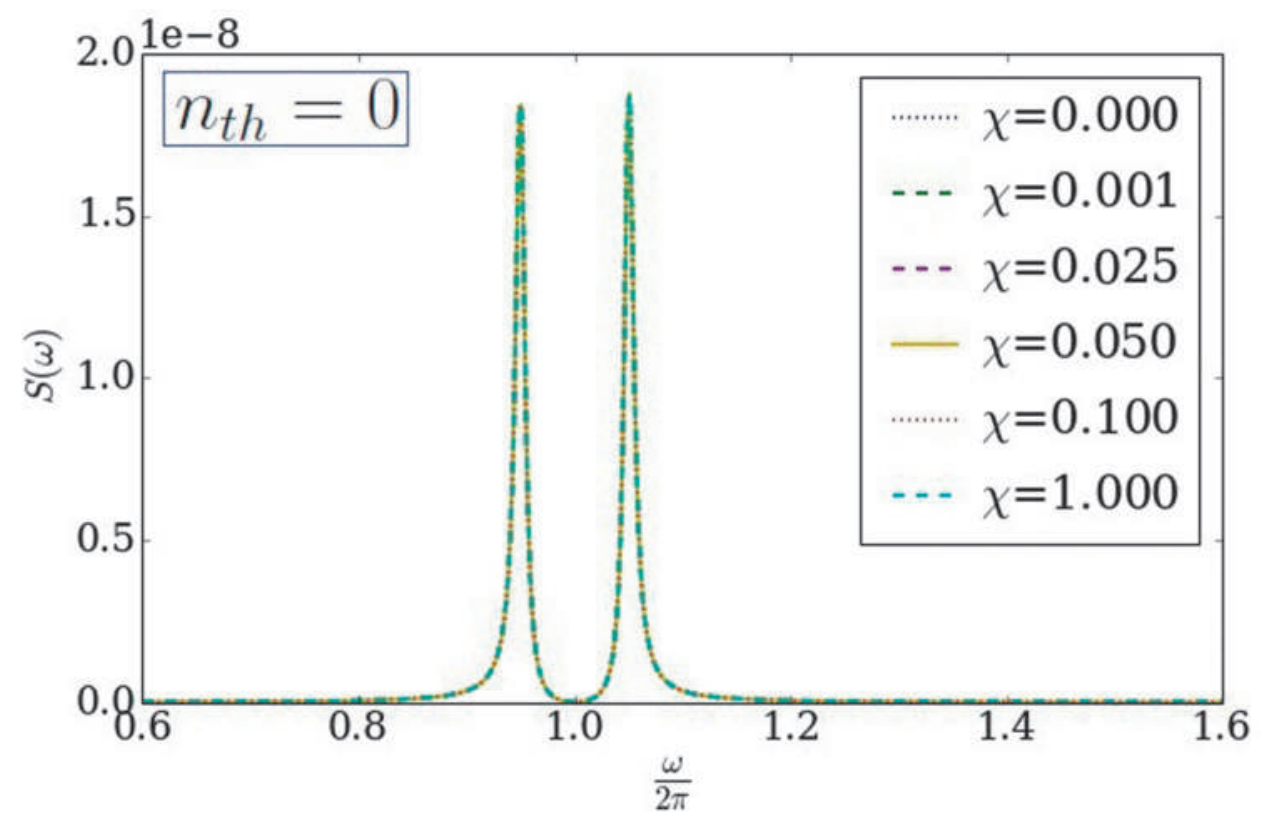

Figura 5. Espectro fotónico en estado estacionario para el modelo Jaynes-Cummings con un medio kerr $\frac{\omega}{2 \pi}=1,0 G H z$, $\frac{\omega_{a}}{2 \pi}=1,0 \mathrm{GHz}, \frac{g}{2 \pi}=0,05 \mathrm{GHz}, \kappa=0,08 \mathrm{GHz}, \gamma=0,05 \mathrm{GHz}, n_{t h}=0$, para diferentes valores de $\chi$. 

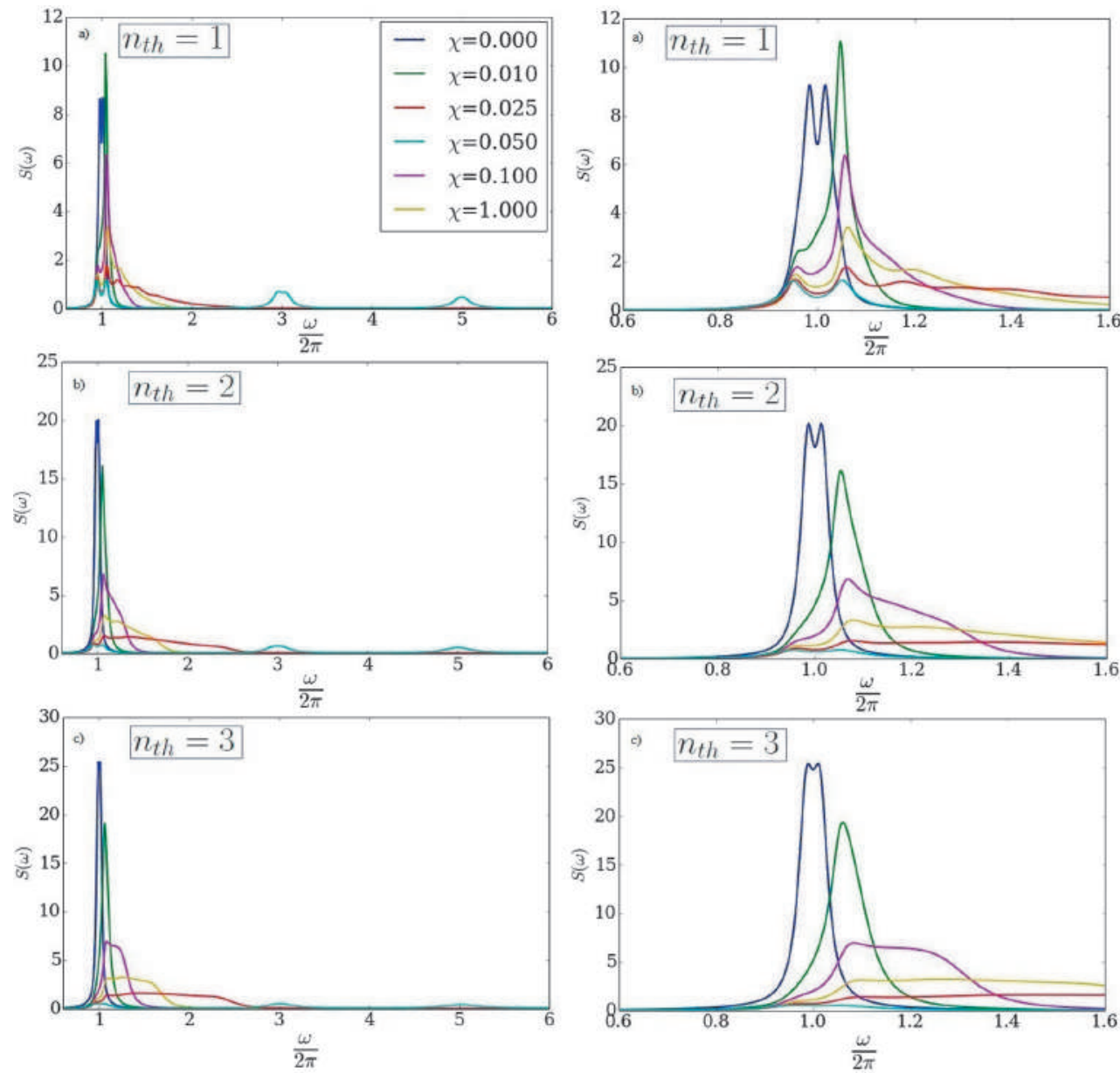

Figura 6. Espectro fotónico en estado estacionario para el modelo Jaynes-Cummings con un medio kerr $\omega=1,0 \times 2 \pi$, $\frac{\omega}{2 \pi}=1,0 \mathrm{GHz}, \frac{\omega_{a}}{2 \pi}=1,0 \mathrm{GHz}, \frac{g}{2 \pi}=0,05 \mathrm{GHz}, \kappa=0,08 \mathrm{GHz}, \gamma=0,05 \mathrm{GHz}$, (a) $n_{t h}=1$,(b) $n_{t h}=2$, (c) $n_{t h}=3$, para diferentes valores de $\chi$.
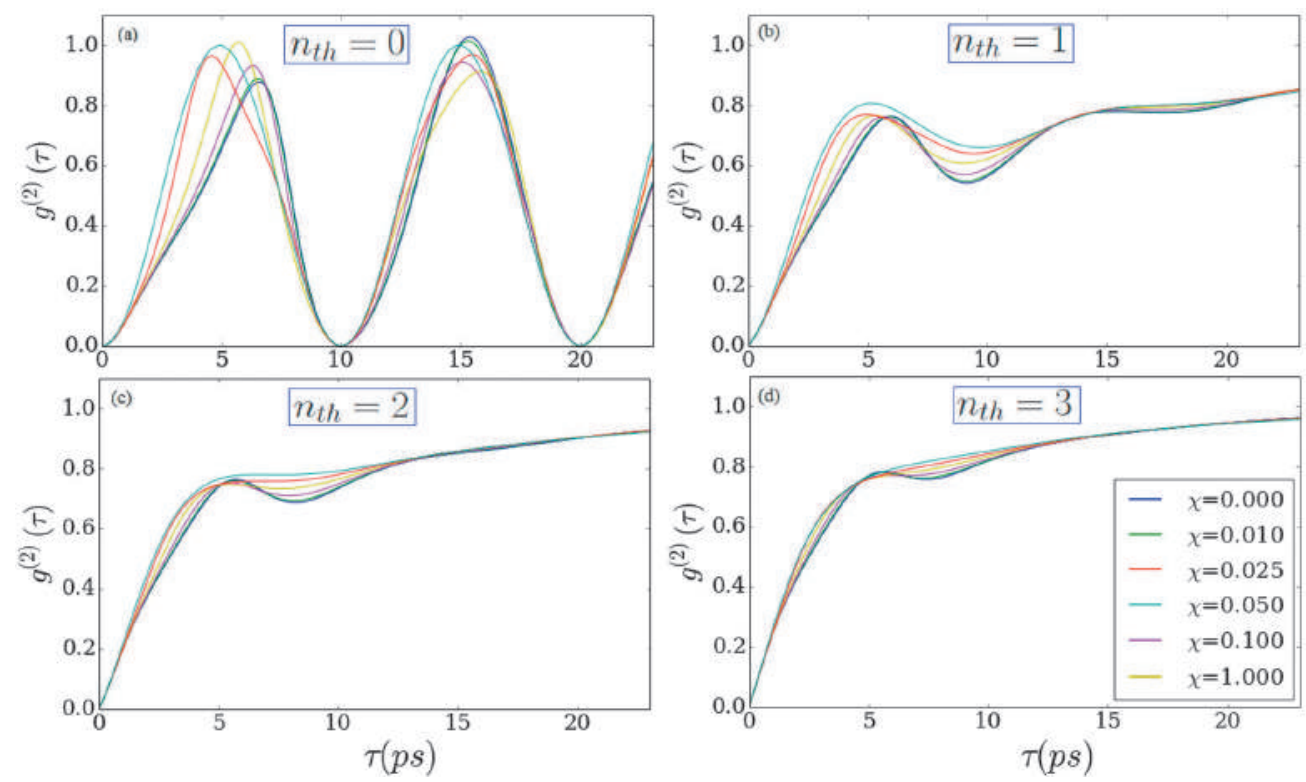

Figura 7. Función de coherencia de segundo orden para el modelo Jaynes-Cummings con un medio kerr $\frac{\omega}{2 \pi}=1,0 G H z$, $\frac{\omega_{a}}{2 \pi}=1,0 \mathrm{GHz}, \frac{g}{2 \pi}=0,05 \mathrm{GHz}, \kappa=0,08 \mathrm{GHz}, \gamma=0,05 \mathrm{GHz}$, (a) $n_{t h}=0$, (b) $n_{t h}=1$, (c) $n_{t h}=2$, (d) $n_{t h}=3$, para diferentes valores de $\chi$. 


\section{CONCLUSIONES}

En este trabajo se han presentado los resultados de una investigación sobre la dinámica de un sistema QED de cavidad donde un solo átomo interactúa con un solo modo del campo electromagnético cuantizado. Para la condición inicial dada y el régimen considerado se encontró el número medio de fotones y la inversión de población $\left\langle\sigma^{\wedge} \sigma^{\wedge}\right\rangle$ del sistema Qubit-Cavidad. En el caso donde no hay disipación se pudo observar que el medio kerr conduce a la disminución de la amplitud de las oscilaciones. Para el caso disipativo, el fuerte acoplamiento genera oscilaciones en el número medio de fotones, debido a que los fotones pasan mayor tiempo en la cavidad antes de ser disipados.

Analizamos analíticamente el comportamiento de la función de correlación excitónica y fotónica de primer y segundo orden sin disipación usando funciones de estados. Para la función de correlación excitónica de segundo orden calculada de forma analítica para los dos modelos, se observó que a medida que $n$ tiende a cero la función de correlación también tiende a ese valor. Como se está trabajando con un medio kerr, hay un proceso de dos fotones y las mínimas condiciónes iniciales que se deben usar es que haya por lo menos un fotón en la cavidad y el Qubit se encuentre en el estado excitado o que el Qubit se encuentre en el estado base y hayan dos fotones en la cavidad.

Para el espectro fotónico notamos en la gráfica que esta función a medida que el número térmico tiende a cero el espectro tiende a ese valor, lo que indica que para estudiar el espectro fotónico se debe tener en cuenta va- lores distintos de cero en el número térmico de fotones asociado con el reservorio, ya que juega un papel fundamental. También se observa que las gráficas de espectros para el modelo de Jaynes Cummings para todos los números térmicos son simétricas con respecto al valor de la frecuencia de la cavidad, lo que concuerda con algunos trabajos teóricos similares reportados. Por último observamos que las funciones de coherencia de segundo orden estudiadas tienen características netamente cuánticas. Por último, esperamos que nuestro estudio pueda proveer suficiente información para futuros estudios teóricos y/o experimentales.

\section{AGRADECIMIENTOS}

Y.R. agradece al profesor Boris Rodríguez de la Universidad de Antioquia por todas las discusiones y sugerencias realizadas en torno a este trabajo.

\section{REFERENCIAS}

[1] E. T. Jaynes and F. W. Cummings, Comparasion of Quantum and Semiclassical Radiation Theories with Application to the Beam Maser. Proc. Inst. Elect. Eng. 51, 89 (1963).

[2] J. Liu and Z.-Y. Li, Interaction of a twolevel atom with single-mode optical field beyond the rotating wave approximation, Opt. Express, Volumen 22, pp. 2867128682 (2014).

[3] M.J.Werner andH.Risken, Quasiprobability dis- tributions for the cavity-damped Jaynes-Cummings model with an additional Kerr medium. Phys. Rev. A 44, 4623 (1991)

[4] O. de los Santos, C. González and J. Récamier, Non- linear Jaynes-Cummings model for two interacting two-level atoms, J. Phys. B: At. Mol. Opt. Phys. Volumen 49, 165503 (2016).

[5] M. Ghorbani, M. J. Faghihi, and H. Safari, Wigner function and entanglement dynamics of a two-atom two-mode nonlinear JaynesCummings model, Jour- nal of the Optical Society of America B, Volumen 34, pp. 1884-1893 (2017).

[6] S.-P. Bu, G.-F. Zhang, J. Liu, Z.-Y. Chen, Berry phase in a two-atom JaynesCummings model with Kerr medium. Phys. Scr. 78, 065008 (2008).

[7] A. Joshi and S.V Lawande, Fluorescence spectrum of a two-level atom interacting with a quantized field in a Kerr-like medium. Phys. Rev. A 46, 5906 (1991).

[8] H. R. Baghshahi, M. J. Faghihi, and M. K. Tavas-soly, Entanglement analysis of a twoatom nonlinear Jaynes-Cummings model 
with nondegenerate two- photon transition, Kerr nonlinearity, and two-mode Stark shift. Laser Phys. Volumen 24, 125203 (2014).

[9] B. Mojaveri, A. Dehghani, M. A. Fasihi and T. Mohammadpour, Thermal Entanglement Between Two Two-Level Atoms in a TwoPhoton Jaynes-Cummings Model with an Added Kerr Medium, International Journal of Theoretical Physics, Volumen 57, pp. 3396-3409, (2018).

[10] D. Vitali, M. Fortunato, and P. Tombesi, Complete Quantum Teleportation with a Kerr Nonlinearity Phys. Rev. Lett. 85, 445 (2000).

[11] A. Joshi and R. R. Puri, Dynamical evolution of the two-photon Jaynes-Cummings model in a Kerr-like médium. Phys. Rev. A 45,5056 (1992).

[12] W. Wei, G. Guang-Can ; Preparation of Nonclassical States of a Cavity Field Based on a Nonlinear Jaynes-Cummings Model. Phys. Rev. Volumen 7, 175 (1998).

[13] E. del Valle, A. Gonzalez-Tudela, F. P. Laussy, C. Tejedor, and M. J. Hartmann. Theory of Frequency-Filtered and TimeResolved N-Photon Correlations, Phys. Rev.Lett. 109,183601 (2012).

[14] E. del Valle, Distilling one, two and entangled pairs of photons from a quantum dot with cavity QED efects and spectral filltering, arXiv:1210.5272v2 [cond-mat.mes-hall] (2012).

[15] H. J. Carmichael, Statistical Methods in Quantum Optics, Vol. 1, Springer-Verlag Berlin Heidelberg, 1999.

[16] J. C. Gonzalez-Henao, E. Plugliese, S. Euzzor, R. Meucci and F. T. Arecchi, Control of entanglement dynamics in a system of three coupled quantum oscillators, Scientific Reports, Volumen 7, 9957 (2017)

[17] J. C. Gonzalez-Henao, E. Plugliese, S. Euzzor, S.F. Abdalah, R. Meucci and J. A. Roversi, Generation of entanglement in quantum parametric oscillators using phase control, Scientific Reports, Volumen 5, 13152 (2015)

[18] J. C. Gonzalez and J. A. Roversi, Decrease of the decay rate of the entanglement of a system of two entangled qubits by increasing the temperature of the thermal bath, Quantum Information Processing, Volumen 14, 1377-1385 (2015).

[19] G. S. Agarwal, Vacuum-field Rabi oscillations of atoms in a cavity, J.opt. Soc. Am.B, Volumen 2, No 3 (1985).

[20] G. S. Agarwal, Probing the dressed states of an atom interacting with a quantized field, Optics Communications, 202-208 (1991).

[21] M. Kronenwett, Photon Correlations in TwoMode Cavity Quantum Electrodynamics, Trabajo de grado para optar el título en Msc en Ciencias físicas Nueva Zelanda, The University of Auckland, 110p, (2007).

[22] S. James Whalen, Photon correlation functions and photon blockade in two-mode cavity QED, Trabajo de grado para optar el título en licenciatura en física, Nueva Zelanda, The University of Auckland, 48p, (2008).

[23] F. P. Laussy, Elena del Valle, and Carlos Tejedor. Luminescence spectra of quantum dots in microcavities. Phys. Rev. B 79, 235325 (2009).

[24] E. del Valle, F. P. Laussy, and C. Tejedor, Luminescence spectra of quantum dots in microcavities. II. Fermions Phys. Rev. B 79, 235326 (2009).

[25] F. P. Laussy, E. del Valle, A. Laucht, J. J. Finley, M. Villas Boas. Luminescence spectra of quantum dots in microcavities. III. Multiple quantum dots, Phys. Rev. B 84, 195313 (2011).

[26] G. S. Agarwal, R. K. Bullow, G. P. Hildred. Exact Finite Temperature quantum statistics of single atom electrodynamics in a cavity of arbitrary Q, Optics Communications, Volumen 59 (1986). 